\title{
Research and Application of Knowledge Management Model Oriented Software Testing Process
}

\author{
Liu Xuemei ${ }^{1,3}$ Gu Guochang $^{1}$ Liu Yongpo ${ }^{2}$ Wu Ji $^{2}$ \\ ${ }^{1}$ College of Computer Science and Technology,Harbin Engineering Univer- \\ sity,Heilongjiang 150001,China; \\ ${ }^{2}$ School of Computer Science and Technology,Beijing University of Aeronautics and As- \\ tronautics,Beijing 100083,China; \\ ${ }^{3}$ Beijing City University,Beijing, 100083,China
}

\begin{abstract}
In software testing organizations, an effective knowledge management of testing process is the key to improve the quality of software testing. Knowledge management has different features in software testing. In this paper, a knowledge management model was proposed towards software testing process, which is based on building the site of knowledge sharing. It emphasizes the idea of closely contacting knowledge service and software testing and will promote the research of knowledge management technology. Simultaneously many key technologies are discussed, such as knowledge representation, knowledge map and etc. At last, an application instance based on this model is given in the project of QESuite2.0.
\end{abstract}

Keywords: Software Testing Process; Knowledge Management Model; Knowledge Sharing; Knowledge Representation; Knowledge Map; Ontology

\section{Introduction}

With the arrival of the age of knowledge economy, the importance of knowledge management that has become a hot spot in international manage fields is more obvious. In order to enhance its competi- tive power, many enterprises have initiatively taken knowledge management into their core business process, which drives the development of consultation business whose new business scope is knowledge management, and then a lot of software tools and systems about knowledge management have been developed by IT enterprise. Magazines and websites which are relative to knowledge management are largely spring up.

The essence of knowledge management is a problem about management, but at last the functions and services of knowledge management are implemented by knowledge management technology. So, many enterprises have difficulties in executing knowledge management effectively without the support of knowledge management, because it is the foundation to construct knowledge management system, and the driving force to implement knowledge management ${ }^{[1]}$.

Broadly, knowledge management technology isn't limited to IT, but modern information technology is the premise to implement knowledge management effectively. For more than ten years of studying knowledge management, many knowledge management IT tools are emerged in commercial market, but not all of them are combined with particular requirement of enterprise, especially in

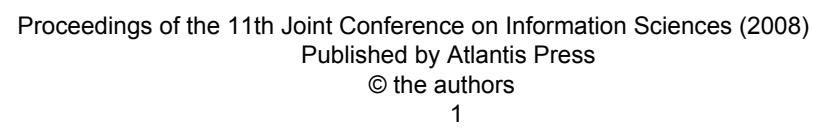


particular fields, the discrepancy between them is highlighted ${ }^{[2]}$.

Software testing is a knowledge-based activity, testing staff are belong to knowledge workers, they test software according to testing plans, but relative knowledge, skills, experiences and inspiration are important to the testing process, if they have no open thoughts, abundant testing experiences and skills, the testing quality can't be assured ${ }^{[3]}$. In addition, developing technology and continually recurring software that will be tested make software testing staff stressed out and prostrated. They have urgent requirements in searching for new testing knowledge and technologies, and that knowledge management offers a new idea and a new method of solving problems for us, but software testing has distinguishing features, although existing multipurpose knowledge management theories and technologies have more or less dealt with many problems, we even more need theories and technologies combined with our field tightly, to survey and deliberate on our problems again, which can help us find an effective method of solving problems ${ }^{[4]}$.

\section{Related works}

At present, knowledge management in the field of software testing is researched seldomly at home and abroad, until now, an example of knowledge management in software testing has not been found. At home research on knowledge management is the latecomer, at the same time, there are little enterprises specializing in software testing, knowledge management has been just executed in testing.

So far, the research and examples of knowledge management in software testing at abroad have not been found, but multipurpose knowledge management in many fields have been researched for many years, such as IBM, Microsoft.
They put in enough money and manpower to research knowledge management, put forward to a suite of theory and developed many software products.

In addition, relative research of knowledge management has been done in software engineering at abroad, a lot of papers have been published and a series of software tools have been developed. Now, KBSE (Knowledge-Based Software Engineering Conference) is held annually, at which the latest advance of the knowledge management in software testing is discussed ${ }^{[5]}$. In fact, research of knowledge management in software testing is an IT problem that the knowledge management ideas are implemented in software testing, that is, a software platform should be developed to support knowledge management activities in software testing. According to the research made by Gallupe in 2000 who studied platforms, theories and examples of existing knowledge management system, there are many difficulties in implementing knowledge acquisition, coding, storage and searching effectively in existing knowledge management platform ${ }^{[6]}$.

\section{Major problems}

According to the working experiences acquired from many software testing projects in BUAA, the author analyzed the testing process of major software with the primary principles of knowledge management, and found that five existing problems in software testing process are:

1.Low ratio of reusing software testing knowledge. At present, the public testing knowledge in software testing process have not been accumulated consciously, although there are some databases about testing knowledge and experiences within business, most staff neglect them, which causes testing knowledge disused and leads to the low rate of testing knowledge and experience.

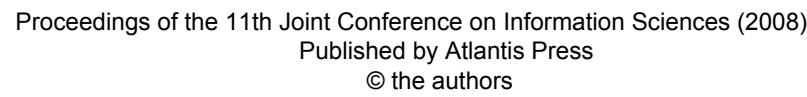


2. Barriers of software testing knowledge transmission. Now the schema to keep testing knowledge makes it very difficult to transmit knowledge, testing knowledge are read by staffs passively. On the other hand, the users always bitterly seek the knowledge they need; the staffs are unable to grasp new testing knowledge fast $^{[7]}$.

3.The poor shared environment of software testing knowledge. There is no shared environment that is formal, special and organized within business, the staffs have few opportunity of mutual interaction. The communication system has not been constructed; the shared knowledge environment of software testing process needs to be further formed.

4. The serious loss of software testing knowledge. Many special experiences and skills are grasped by only a few people, which don't really become public knowledge. Not only this causes many difficulties in transmitting testing knowledge, but also a turnover of staff will cause loss of testing knowledge and complete competitiveness fluctuations, which will cause enterprises to incur serious losses $^{[8]}$.

5. Impossible to achieve the most optimum distribution of human resources fast. Knowledge management is integration with human, process and technology, among which human is the most important part. If the enterprise managers can't know the staff's specialty and knowledge very well, the optimal team will not be organized in a new testing project, so the most optimum distribution of human resources can't be achieved.

The above-mentioned problems cause inefficient software testing, slow response to the market, weak strain capacity. The author thinks that no scientific knowledge management in software testing leads to emerge above-mentioned problems, so it is very important to carry out knowledge management in this field.

\section{Knowledge management model of software testing process}

The essential object of knowledge management is to promote knowledge reusing, and not simply knowledge accumulation or store. To promote knowledge reusing, the transmission of knowledge is based on knowledge recognition, whose main feature is to offer decision support information in the activities for staffs.

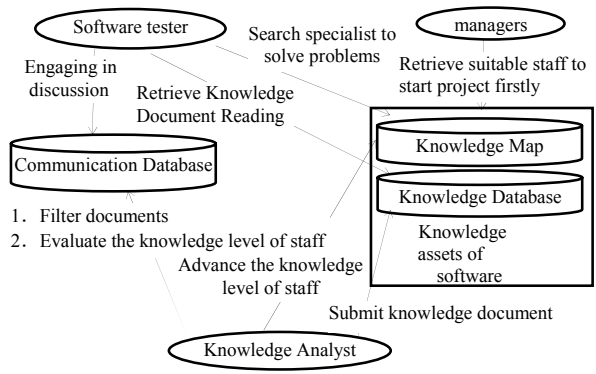

Figure 1 Knowledge Management Model

In knowledge management of software testing, initiative transmission of knowledge must be implemented to establish channels of communication between organized staff. According to a need for knowledge in software testing, correlative transmission of knowledge can be achieved in time; the effect of reusing knowledge can also be advanced by using effective transmission of knowledge. Because the knowledge is always changing dynamically, an effective infrastructure must be built to meet above-mentioned functions, so a knowledge management model oriented software testing process is proposed, as shown figure 1 .

The element of this model are testing organization staffs, testing organization knowledge assets, testing organization communication site. The idea of this model is to build a communication site where the problems raised by staffs, the solving process and documents are recorded. By documents filtering, the appropriate document submission knowledge analyst who can decide if the docu-

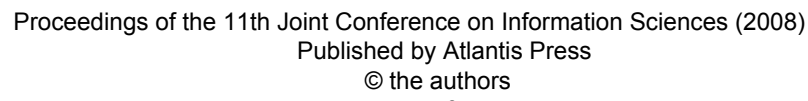


ment is submitted to knowledge database will be found.

Knowledge analyst can also promote the knowledge level of staffs according to the knowledge documents published by staffs in knowledge database. Other testing staffs may retrieve information in knowledge document database to find documents that can solve problems when they come across problems. If they can't find appropriate documents, they can discover specialists who can solve problems in terms of the problem classification. The organization managers can rapidly find appropriate staffs to start a project by knowledge map on basis of the project requirements.

\section{Knowledge representation}

The software testing is an intensive knowledge process driven by testing knowledge that are grasped by testing staffs. The knowledge relative to the software testing process are within the limits of software testing knowledge management. In the knowledge management system, persons are the users of knowledge, the usage mode is reading and comprehension, the problems will be solved by authors themselves. The expression of knowledge should be easy to be used, read and comprehended, so natural language is the most appropriate ${ }^{[10]}$. In order to make staffs easy to use and comprehend knowledge, the software testing knowledge are represented by ontology.

\subsection{Ontology}

Ontology which derived from philosophy is about existence and essence. Newly it is used as a method of knowledge representation, knowledge-sharing and reused in computer modeling. In 1993, a popular conception of ontology was proposed by Gruber, this is, an ontology is an explicit specification of a conceptual model. An ontology theory is an explicit expression and description of conceptual objects, and is also systematized description of external reality. Essentially, an ontology is a concept of one or some fields and a set of relations among these concepts. The relation shows the restrict and connection of the concepts, but the relation itself is also a concept, new relations may be constructed between relations.

\subsection{Ontology-based knowledge repre- sentation}

In knowledge representation of software testing, relative concepts, attributes and relations are described by using ontology. There are five ontologies in the system; they are documents, references, projects, staff and knowledge level, as shown in figure 2 .

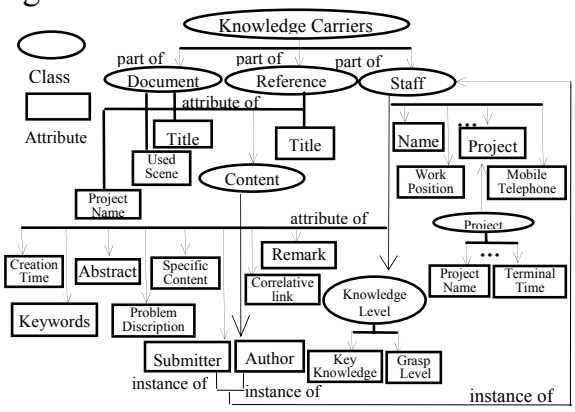

Figure 2 Knowledge Ontology

\subsection{Ontology-based search and sort- ing of knowledge documents}

Ontology mainly acts as knowledge database in knowledge retrieval subsystem. It classifies concepts of software testing, describes relation restriction between concepts, and constructs detailed knowledge database which involves concrete concepts, attributes, relations, and instances between concepts. When retrieving knowledge, the correlative concepts or attributes are found according to the users' requests. Start from there, the ontology information will be checked to see if it is related to the concepts or attributes. Logically intelligent retrieval is then achieved. 
After retrieving, we need to find out how to organize the retrieved documents in a sequential manner. Many factors can influence the sorting process. According to our research, there are five key factors that could influence the sorting results, such as users' evaluations of documents, knowledge analysts' evaluation of documents, users' knowledge level, the number of linking documents and the number of opening documents. The weights of the five factors are in descending order. Every factor weight is calculated by using descending weight formula. The formula is as follow:

$$
\mathrm{P}_{\mathrm{s}}=2(\mathrm{~N}-\mathrm{S}+1) / \mathrm{N}(\mathrm{N}+1)
$$

Where $\mathrm{S}$ is parameters sorting, $\mathrm{N}$ is parameter numbers, and $\sum \mathrm{Ps}, \mathrm{s}=1$ to $\mathrm{N}=1$.

While $\mathrm{N}=5, \mathrm{P}_{1}=0.33, \mathrm{P}_{2}=0.27, \mathrm{P}_{3}=0.2$, $\mathrm{P}_{4}=0.13, \mathrm{P}_{5}=0.07, \sum \mathrm{Ps}, \mathrm{s}=1$ to $5=1$.

The importance of knowledge document is calculated as follows:

The importance of knowledge document $=\mathrm{P}_{1} \times$ users' evaluation of document

$+\mathrm{P}_{2} \times$ knowledge analysts' evaluation of document $+\mathrm{P}_{3} \times$ users' knowledge level $+\mathrm{P}_{4} \times$ the number of linking document + $\mathrm{P}_{5} \times$ the number of open document.

According to the importance results, all documents will be in descending order, then the most valuable knowledge document will be on the top.

\section{Constructing knowledge map}

\subsection{Definition}

Knowledge map (called as knowledge yellow pages) is a stock catalogue about knowledge. The knowledge source shown by knowledge map could be department name, team name, specialist's name, related person's name, filename, bibliography, event number, patent number, or knowledge database index. However, the knowledge content is not included. It is a guide to save time in tracing the knowledge source.

\subsection{Conditions of constructing knowledge map}

When there is a good knowledge map, whatever deserted the needed knowledge is, by tracing correlative information, a fountain of knowledge will be found as long as making the running. By this snowball effect, staffs can't devote too much time to acquire correlative knowledge when they need knowledge. The organization can find out what knowledge will be enhanced or developed and scattered by using knowledge map.

In order to construct a good knowledge map, five works must be achieved.

1. To classify the status of important knowledge and skills.

2. To distinguish the level of knowledge and skills.

3. To decide the category and level of knowledge needed by specific post.

4. To evaluate ability of staff who has knowledge.

5. To build a knowledge map retrieval system.

\subsection{Knowledge level and evaluation standard of software testing knowledge}

A good software testing knowledge management platform should also offer strong software testing knowledge classification. According to the practical experience and SWEBOK methods ${ }^{[12]}$, five knowledge fields are added into software testing. They are developing language, database, operating system, software testing tools, related knowledge for the testing project. In this knowledge map, the knowledge level is divided into five levels in each filed. They are comprehension, acquaintance, mastership, conversance, and specialist. Definition for each level is clearly described and easily to be evaluated. Every employee' ability is evaluated by this criteria. The evaluation process should be done by the employee, team, manager, and knowledge analyst coopera-

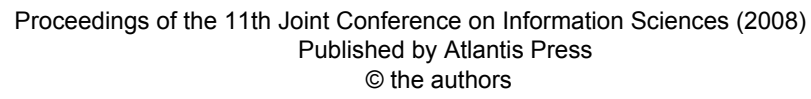


tively. The definitions of five levels of knowledge are given as follows:

1. Comprehension. Staffs has used the technology for half a year and taken part in a project. They can reference technology documents or help files to need their requirements, know the effect of the technology.

2. Acquaintance. Staffs can grasp more than $50 \%$ of key technology and has used it for a year and taken part in more than two projects by using it.

3. Mastership. Staffs can grasp more than $60 \%$ of key technology and has used it for two years and taken part in more than three projects by using it.

4. Conversance. Staffs can grasp more than $85 \%$ of key technology and has used it for five years and taken pare in more than five projects by using it.

5. Specialist. Staffs can grasp more than $95 \%$ of key technology and has used it for seven years and taken part in more than eight projects by using it.

According to our research, when evaluating staffs knowledge levels, the top three levels can be upgraded automatically in terms of staffs' working experience and utility time. But when staffs will be evaluated as conversance or specialist, the utility time and projects which are taken part in by them are only necessary conditions. Even if they meet the conditions, they are not always to be evaluated as conversance or specialist, their levels must be customized by knowledge analyst by hand.

\section{Case study}

The present system is a sub-system of QESuite2.0, which is a software testing management platform developed by Beijing University of Aeronautics and Astronautics (BUAA). By now, a proto-system has been built. All the modules and functions have been implemented. This system is based on the above-mentioned knowledge management map and has been used in actual work with anticipated results.

According to the knowledge classification and staffs' knowledge levels, a specialist network is built. The knowledge documents can be filtered by discussion in communication database. The knowledge documents will be integrated into knowledge database to accumulate knowledge by using knowledge classification trees. Knowledge retrieval engine makes users easy to retrieve required knowledge. When users can't find appropriated results, this system will tell who know the answers, so that knowledge sharing can be implemented. For the sake of data security, many access roles and access permission have been defined ${ }^{[13]}$.The architecture of this system is shown as Figure 3.

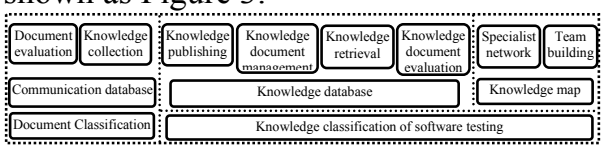

Figure 3 Architecture

The system is based on J2EE and is a knowledge management platform oriented software testing process. The system uses the knowledge life-cycle management as a guide ${ }^{[14]}$. It can help enterprises to store, manage, search, and share all kinds of knowledge by using the knowledge documents. It can evaluate the knowledge level of staff by using the knowledge map. Using knowledge map will become a symbol for intelligent staff. The system will affirm the staff who has knowledge by statistics, and that will improve the culture of knowledge-sharing in the enterprise. The workflow is shown as Figure 4. 


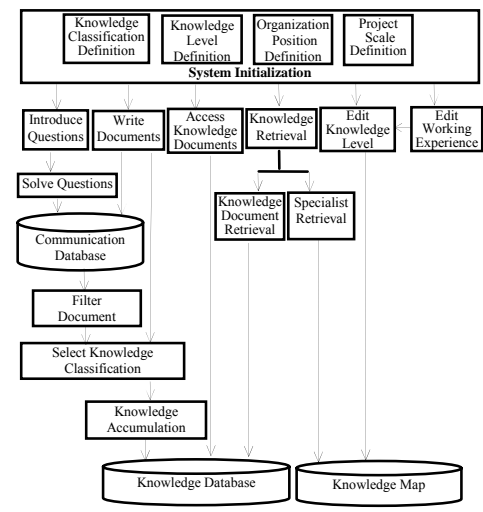

Figure 4 Workflow Diagram

\section{Conclusion}

The emergence of knowledge management offers a new idea and method to solve problems for us, but software testing has its features. The present theories and technologies of common knowledge management have more or less touched some problems, but we much more need theories and technologies closely correlative to software testing to think about and survey our problems again, so that a more effective method will be found. Research of knowledge management in software testing is very important to advance the testing level and strain capacity, improve the quality of software products and the economic benefit, and promote the nuclear competitive power of software enterprise.

Software testing technologies, knowledge management and knowledge management model are discussed in this paper. On the basis of systemically summary of these fields, we analyzed the knowledge service model, and then constructed knowledge management model oriented software testing. At last this system is designed and implemented which is the basis of further investigations of knowledge management.

\section{Acknowledgements}

Liu Xuemei was supported by the project "Research of analyzing and diagnosing the performance of server software based on data mining" funded by national natural science.

\section{References}

[1] SEIJA K S, Annukka M, Veikko S. Toward a Practical Solution for Capturing Knowledge for Software Projects[C]. IEEE Software, May/June, 2002: 60-62

[2] Microsoft. Digital Nervous System -Practical Knowledge Management. Microsoft White Paper [Z]. Beijing: Microsoft(China) Limited Company, 1999.

[3] Ioana Rus, Mikael Lindvall, and Sachin Suman Sinha, Knowledge Management in Software Engineering A State-of-the-Art-Report, The University of Maryland, 2001

[4] George Lawton. Knowledge Management: Ready for Prime Time? IEEE Computer, 2001, 34(2): 12-14

[5] Arik Johnson. An introduction to knowledge management as a framework for competitive intelligence. [White Paper]. International Knowledge Management Executive Summit, 1998, California

[6] R. Brent Gallupe, "Knowledge Management Systems: Surveying the Landscape", Queen's University at Kingston, October 2000

[7] loana Rus, Mikael Lindvall. Knowledge Management in Software Engineering. IEEE Software, May/June 2002, pp. 26-38

[8] Gerhard Fischer, Jonathan Ostwald. Knowledge Management: Problems, Promises, Realities, and Challenges. IEEE Intelligent Systems, January/February, 2001:60-72

[9] Nakkiran N Sunassee, David A Sewry. A theoretical framework for knowledge management implementa-

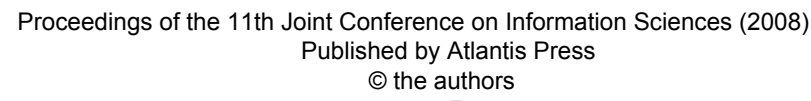


tion. In: Proc of the SAICSIT 2002

ACM International Conference Proceeding Series, 2002. 235-245

[10] Kurt Schneider, Jan-Peter von Hunnius, Victor R. Basili. Experience in Implementing a Learning Software Organization. IEEE Software, May/June 2002: 46-49

[11] MAYER G J. The art of software testing[M], John Wiley, New York, 1979,35-37.

[12] Software Engineering Body of Knowledge 2004 Version[M] ,IEEE

[13] HE Zhitao. Research and Implement of Knowledge Management System for Software Testing Process(D). Beijing: Beijing University of Aeronautics and Astronautics, 2003.

[14] Pravin V.Tulachan. The Development Guide of EJB Components[M]. Beijing: Tsinghua University publishing company, 2002. 